\title{
Salmonellosis en el lactante. Análisis de 93 casos hos pitalizados en Hospital Regional Leonardo Guzmán de Antofagasta
}

Dres. Paulino Guerra A. *, Manuel Zamorano G. **, Manuel Yáñez Z.**

Las infecciones por Salmonellas revisten importancia creciente en los hospitales pediátricos. Esto deriva por un lado por la frecuencia con que se producen verdaderos brotes epidémicos. por la distinta naturaleza de los cuadros en el lactante y por la dificultad de tratamiento específico $^{1-2-3-4-5-\gamma}$

Así, tenèmos que de la localización enteral es posible la generalización con compromiso cerebro-meníngeo, osteoarticular, de abscesos, etc., agravando considerablemente el pronóstico $^{6.7}$. Esta situación es particularmente grave en el lactante menor de seis meses, disminuyendo progresivamente con la edad. Referente a la diferenciación microbiológica en los últimos años se ha recomendado la clasificación en Salmonella Cholera Suis, Salmonella tiphy y Salmonella enteritidis. A esta última pertenecen los bioserotipos más frecuentes en nuestro medio, especialmente la variedad Tiphymurium $^{9-12}$. Sin embargo esta nomenclatura no se utiliza en todos. los establecimientos, lo que lleva a la confusión.

* Servicio de Pediatría, Hospital Regional Leonardo Guzmán.

** Departamento Ciencias de la Salud. Universidad de Chile - Sede Antofagasta.
Se estima que las formas enterales evolu. cionan bien sin tratamiento antibiótico $y$ asi es menor la frecuencia del estado de portador, no obstante en los menores de seis meses la mayor gravedad de estos cuadros obliga a utilizarlos, basándose en el antibiograma habitual del lugar est udiado.

Esto se recomienda por la distinta sensibilidad observada en diversos estudios, lo que hace difícil recomendar un solo esquema terapéutico $^{5}$.

Por último, debe tenerse siempre presente el aspecto epidemiológico y ante un brote estudiar detenidamente la vía de infección y sus mecanismos de contagio, como material de aseo, vectores, alimentos, etc. ${ }^{10}$.

\section{MATERIAL Y METODO}

Se analizaron 93 historias clínicas de lactantes hospitalizados en el Servicio de Pediatría del Hospital Regional Leonardo Guzmán de Antofagasta, desde enero 1973 a diciembre 1978, con el diagnóstico de Salmonellosis. Requisito de ingreso a esta casuística fue tener bacteriología positiva. No se incluyeron los 
casos de Tifoidea. Se estudiaron los siguientes aspectos:

- Edad, sexo y estado nutritivo.

- Incidencia estacional.

- Diagnóstico de ingreso.

- Características clínicas.

- Recuento leucocitario.

- Identificación bacteriológica.

- Letalidad.

- Evolución.

- Tratamiento.

\section{RESULTADOS}

En la Tabla 1 podemos observar que la mayor incidencia se presentó bajo el $6^{\circ}$ mes, con 48 casos $(51,6 \%)$ y de 6 a 11 meses 36 casos $(38,7 \%)$, lo que hace un $90,3 \%$ bajo el año de edad. No hubo diferencias significativas en cuanto al estado nutritivo, ya que se presentó en 52 normales $(55,9 \%)$ y 41 desnutridos $(44,1 \%)$. Debe sí destacarse que la totalidad de los casos sobre el año fueron desnutridos. En cuanto al sexo hubo predomin io masculino con 57 casos $(61,3 \%)$.

Tabla 1

DISTRIBUCION POR EDAD, SEXO Y ESTADO NUTRITIVO DE 93 LACTANTES CON SALMONELLOSIS. (HOSPITAL REGIONAL DE ANTOFAGASTA)

\begin{tabular}{|c|c|c|c|c|c|c|}
\hline \multirow{3}{*}{ Grupo etario } & \multicolumn{4}{|c|}{ Estado nutritzoo } & \multirow{3}{*}{$\begin{array}{l}N^{\circ} \text { de } \\
\text { casos }\end{array}$} & \multirow{3}{*}{$\%$} \\
\hline & \multicolumn{2}{|c|}{ Eutrófico } & \multicolumn{2}{|c|}{ Desnutrido } & & \\
\hline & 9 & \& & $q$ & 。 & & \\
\hline $28 \mathrm{ds} .-5$ meses & 14 & 16 & 8 & 10 & 48 & 51,6 \\
\hline $6-11$ meses & 3 & 14 & 6 & 13 & 36 & 38,7 \\
\hline $12-17$ meses & 0 & 2 & 3 & 0 & 5 & 5,4 \\
\hline $18-23$ meses & 1 & 2 & 1 & 0 & 4 & 4,3 \\
\hline Total & 18 & 34 & 18 & 23 & 93 & 100,0 \\
\hline
\end{tabular}

Hallazgos bacteriológicos: Como se aprecia en la Tabla 2, en 40 casos (43\%) se aisló Salmonella Sp. sin mayor diferenciación. EJ serotipo más frecuente fue tiphymurium en 24 casos $(26,9 \%$ ).

En la Tabla 3 se señala el origen de la muestra. En 80 casos sólo se aisló el germen de la de-

Tabla 2

\section{IDENTIFICACION BACTERIOLOGICA EN 93 CASOS DE SALMONELLOSIS. (hospital Regional)}

\begin{tabular}{lcc}
\hline \multicolumn{1}{c}{ Ident. } & Ident. $\begin{array}{c}\text { bacteriológica } \\
N^{0} \text { decasos }\end{array}$ & $\%$ \\
\hline Salmonella Sp. & 40 & 43,0 \\
S. enteritidis grupo B & 23 & 24,7 \\
S. Tiphymurium & 25 & 26,9 \\
S. grupo $A$ & 4 & 4,3 \\
S. grupo $C$ & 1 & 1,1 \\
Total & 93 & 100,0 \\
\hline
\end{tabular}

posición. En 7 hubo hallazgo en el coprocultivo y además en otras muestres (hemocultivo 4, LCR y LSD 1, pus absceso 1, urocultivo 1). En 6 casos se aisló el agente en la sangre, resultando las muestras de deposiciones negativas.

Tabla 3

ORIGEN DE MUESTRAS POSITIVAS EN 93 LACTANTES CON SALMONELLOSIS. HOSPITAL REGIONAL DE ANTOFAGASTA

\begin{tabular}{lrr}
\hline Muestras & \multicolumn{2}{c}{$N^{\circ}$} \\
& casas & \multicolumn{1}{c}{$\%$} \\
\hline Cprocultivo & 80 & $\mathbf{8 6 , 0}$ \\
Coprocultivo + hemocultivo & 4 & 4,3 \\
Coprocultivo + localizaciones secundarias & & \\
(LCR, LSD, pus absceso, orina) & 3 & 3,2 \\
Hemocultivo & 6 & 6,5 \\
Total & 93 & 100,0 \\
\hline
\end{tabular}


Incidencia estacional: Su distribución se estudia en la Tabla 4. Es llamativa la baja frecuencia observada durante el invierno con sólo 7 (asos $(7,5 \%)$.

Diagnóstico de ingreso y evolución: El diagnóstico de ingreso se considera en la Tabla 5 .

Se aprecia que el diagnóstico más frecuente fue el de Síndrome Diarreico Agudo, que se consignó en 56 casos $(60,2 \%)$; las Infecciones

Tabla 4

INCIDENCIA ESTACIONAL EN 93 LACTANTES CON SALMONELLOSIS HOSPITAL REGIONAL ANTOFAGASTA

\begin{tabular}{lcc}
\hline Estación & $N^{\circ}$ casos & $\%$ \\
\hline Otoño & 36 & 38,7 \\
Primavera & 21 & 22,6 \\
Verano & 29 & 31,2 \\
Invierno & 7 & 7,5 \\
Total & 93 & 100,0 \\
\hline
\end{tabular}

Tabla 5

DIAGNOSTICO DE INGRESO EN 93 LACTANTES CON SALMONELLOSIS

\begin{tabular}{lcc}
\hline Diagnóstico & $\begin{array}{c}N^{\circ} \text { de } \\
\text { casos }\end{array}$ & $\%$ \\
\hline Síndrome diarreico agudo & 56 & 60,2 \\
Infecciones respiratorias ag. & 18 & 19,4 \\
Otros & 19 & 20,4 \\
\cline { 2 - 3 } & 93 & 100,0 \\
\hline
\end{tabular}

Respiratorias Agudas se presentaron en 18 casos (19.4\%). En el rubro Otros se anotaron diagnósticos como Meningitis (3), Sepsis (2), Estado Infeccioso (5) y patología variada en el resto, alcanzando a 19 casos en total $(20,4 \%)$.

Cabe consignar que 31 casos $(33,3 \%)$ fueron de origen intrahospitalario.

EJ promedio de hospitalización fue de 28,7 días, los promedios de duración de la fiebre, alteración de deposiciones y mantención

Gráfico 1

FRECUENCIA DE SIGNOS Y SINTOMAS EN 93 CASOS CON SALMONELLOSIS HOSPITAL REGIONAL, ANTOFAGASTA

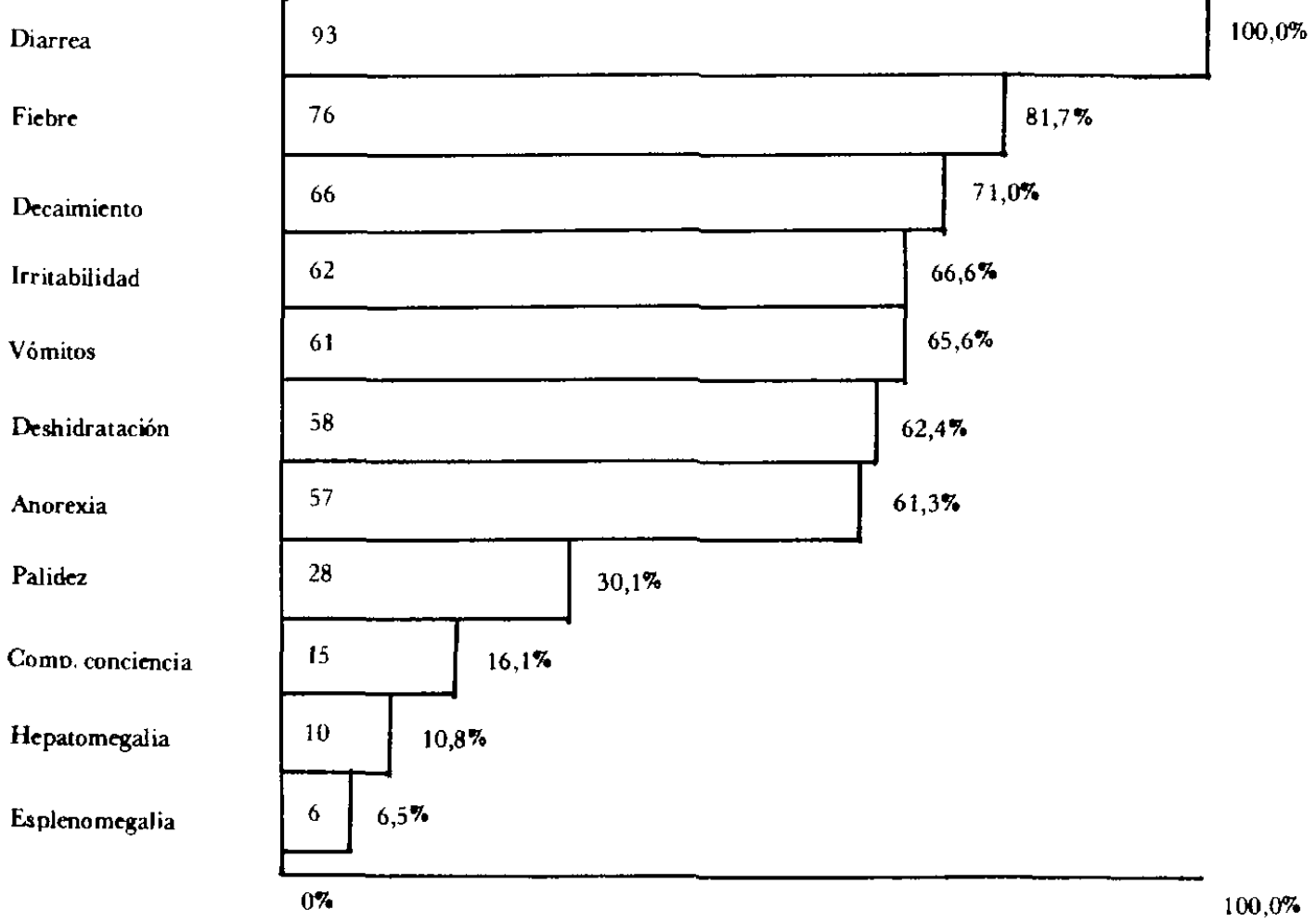


de la fleboclisis fue de 5,$5 ; 17,6$ y 4,3 días respectivamente.

Cuadro clánico. Se analiza en el Gráfico 1, en el que se ubican en orden decreciente de frecuencia los distintos hallazgos semiológicos. La diarrea se presentó en el $100 \%$ de los casos y en $30(32,3 \%)$ fue francamente enterocólica; la fiebre en el $81,7 \%$ y la deshidratación en el $62,4 \%$. La hepatomegalia sólo estuvo presente en 10 enfermos $(10,8 \%)$ y la esplenomegalia en $6(6,5 \%)$.

Recuento Leucocitario. De acuerdo al criterio de Derman el recuento fue normal en $63 \mathrm{en}$ fermos $(87,5 \%)$; hubo leucocitosis en $6(8,3 \%)$ y leucopenia en $3(4,2 \%)$. En 21 pacientes no se realizó este examen.

Tratamiento antibiótico. Se controló el empleo de antibióticos en 83 pacientes, seleccionándose generalmente en base al antibiograma o al estudio de sensibilidad de la cepa origen del brote en el Servicio. La falta de respuesta clínica obligó al empleo sucesivo o simultáneo de uno o más antibióticos. La vía, dosis y duración del tratamiento fue la habitual. Este análisis se hace en el Gráfico 2, en que se estudian en orden de frecuencia decreciente el número de casos en que se empleó cada antibiótico. El promedio de antibióticos por enfermo fue de 2,8 .

Gráfico 2

\section{ANTIBIOTICOS EMPLEADOS EN 83 PACIENTES CON SALMONELLOSIS} HOSPITAL REGIONAL, ANTOFAGASTA

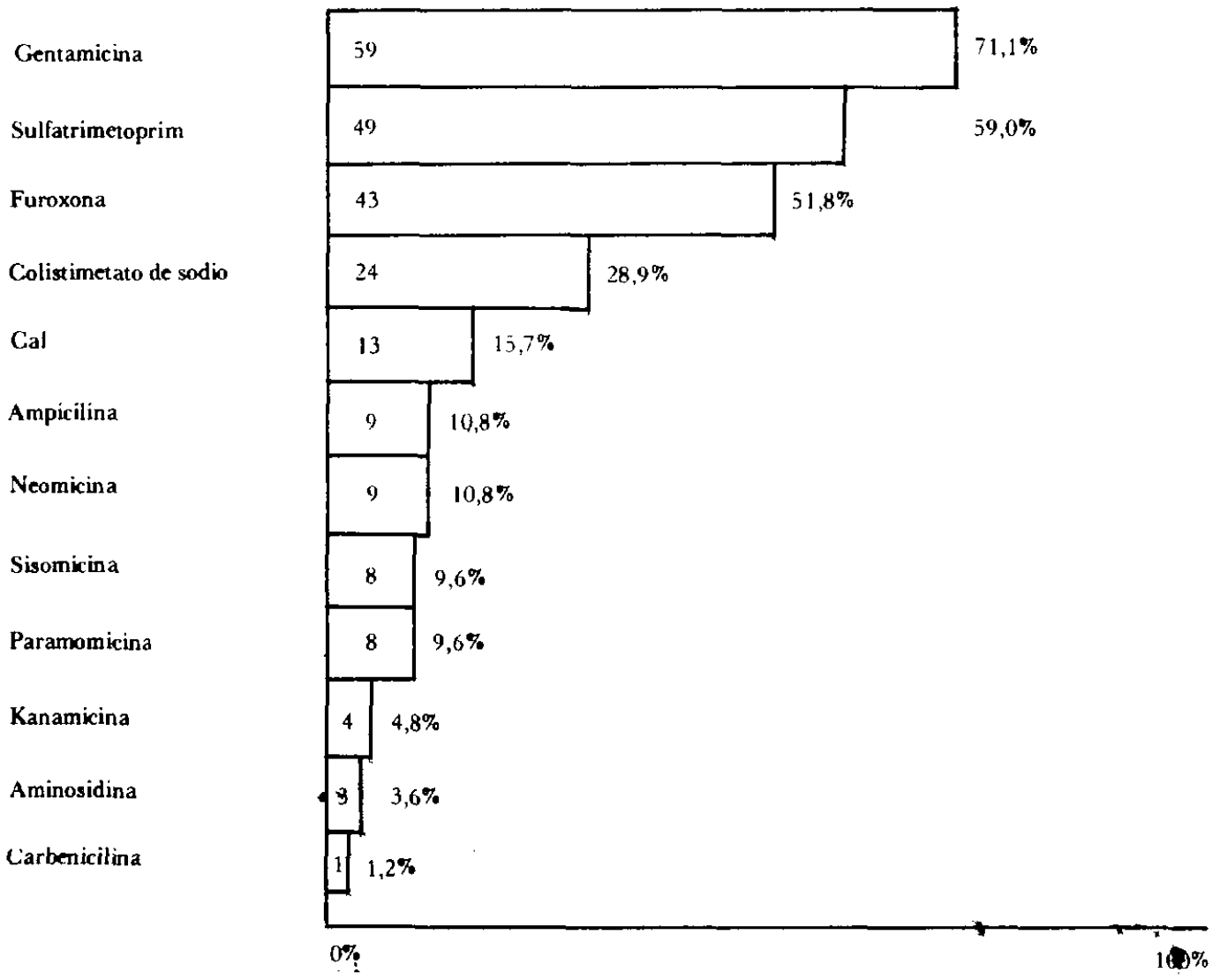


Letalidad. En la Tabla 6 se estudian los casos fallecidos de acuerdo a su edad, sexo y est ado nutritivo. El total alcanzó a 8 enfermos, lo que da un $8,6 \%$. Destaca que el $100 \%$ era menor de seis meses de edad. No se encontraron diferencias en desnutridos y eutróficos o según sexo.

Tabla 6

LETALIDAD SEGUN SEXO Y ESTADO NUTRITIVO EN 93 PACIENTES CON SALMONELLOSiS. HOSPITAL REGIONAL, ANTOFAGASTA

\begin{tabular}{|c|c|c|c|c|c|c|}
\hline \multirow{2}{*}{$\begin{array}{c}\text { Edad en } \\
\text { meses }\end{array}$} & \multicolumn{2}{|c|}{ Sexo } & \multicolumn{2}{|c|}{ Estado nutritivo } & \multirow{2}{*}{$\begin{array}{l}\mathrm{N}^{\circ} \text { de } \\
\text { casos }\end{array}$} & \multirow[b]{2}{*}{$\%$} \\
\hline & 8 & 8 & Desnut. & Normales & & \\
\hline $1-5$ & 5 & 3 & 4 & 4 & 48 & 51,6 \\
\hline $6-11$ & - & - & - & - & 36 & 38,7 \\
\hline $12-17$ & - & - & _- & - & 5 & 5,4 \\
\hline $18-23$ & - & - & - & - & 4 & 4,3 \\
\hline Total & j & 3 & 4 & 4 & 93 & 100,0 \\
\hline
\end{tabular}

La letalidad según forma clínica se considera en la Tabla 7 . Hubo igual número de fallecidos (4) en las formas enterales y sistémicas, sin embargo la letalidad en esta última condición alcanzó al 28,6\% sobre el 5,1\% de las formas enterales. En los cuatro fallecidos con compromiso sistémico hubo meningitis.

\section{Tabla 7}

LETALIDAD SEGUN FORMA CLINICA EN 93 CASOS CON SALMONELLOSIS HOSPITAL REGIONAL, ANTOFAGASTA

\begin{tabular}{lccc}
\hline Forma & $N^{\circ}$ casos & Fallecidos & Letalidad \\
\hline Enteral & 79 & 4 & 4,3 \\
Sistémica & 14 & 4 & 4,3 \\
\hline Total & 93 & 8 & 8,6 \\
\hline
\end{tabular}

También podemos destacar que 4 casos fallecidos procedieron de hospitales de fuera de Antofagasta, lo que hace una letalidad, para este grupo de niños, mayor que la correspondiente a los pacientes de esta ciudad. Esto lo hemos relacionado con la tardanza en el estudio bacteriológico y la iniciación de un tratamiento mejor dirigido.

\section{COMENTARIO}

Las salmonellosis representan un problema de difícil manejo en los servicios hospitalarios de
Pediatría. Esto deriva de la variedad del cuadro clínico, de las dificultades de diagnóstico de laboratorio, de la frecuente aparición de cepas resistentes y de problemas en el control epidemiológico.

Así en el lactante menor de seis meses es más frecuente que ocurra enfermedad clínica en caso de infección y es también más frecuente que la localización gastrointestinal pueda extenderse con bacteremia con focos a distancia, especialmente meníngeos y articulares.

Las dificultades de diagnóstico de laboratorio de un hospital regional derivan del gran número de serotipos que a la vez son agrupados de distinta manera por los bacteriológicos. La falta de sueros muy purificados, caros y específicos hace problemática una adecuada identificación y uno se pregunta si realmente se justifica la creación de tantos serotipos apenas distintos (más de 1.000 en la actualidad).

En cuanto el tratamiento es conocido el diferente enfoque de las formas enterales de las sistémicas; mientras en las primeras es recomendable no usar antibióticos, ya que no acelera la recuperación y además aumenta el número de portadores crónicos, en las segundas el pronóstico depende del tratamiento oportuno y adecuado. En este sentido es útil conocer un antibiograma actualizado de las cepas habituales de los distintos servicios hospitalarios que sirven de 
orientación para el inicio del tratamiento, el que podrá adecuarse de acuerdo al antibiograma y/o respuesta in vivo observada.

Por último, cuando nos enfrentamos a un brote epidémico en un servicio determinado, no ha de bastar el tratamiento de los casos denunciados, sino que ha de estudiarse toda la cadena epidemiológica, incluyendo posibles vectores como moscas, baratas; vehículos como material de aseo, instrumental, etc.; posibles portadores como personal de SEDILE, etc., sin olvidar que a nivel externo las salmonellas se encuentran en una gran variedad de animales domésticos, como perros, gatos, tortugas, gallinas, etc. y otros como ratas y cerdos, lo que naturalmente dificultará el control de esta patología infecciosa.

\section{RESUMEN}

Se analizaron 93 historias clínicas de lactantes hospitalizados desde enero 1973 a diciembre 1978 en el Servicio de Pediatría del Hospital Leonardo Guzmán de Antofagasta, con el diagnóstico de Salmonellosis. No se incluyeron los casos con desarrollo de Salmonella Tiphy

La mayor incidencia se tuvo bajo los 6 meses, con 48 casos $(51,6 \%)$ y de 6 a 11 meses 36 casos $(38,7 \%)$, lo que suma $90,3 \%$ bajo el año de edad. No se observó diferencias en cuanto al estado nutritivo y hubo un predominio del sexo masculino, con 57 casos $(61,3 \%)$.

En 40 enfermos sólo se identificó Salmonella sp sin mayor diferenciación. 23 casos $(24,7 \%)$ corresponden a Salmonella Enteritidis grupo B y 25 a la variedad tiphymurium $(26,9 \%) .80$ pacientes tuvieron hallazgo de la salmonella en el coprocultivo. En 10 casos fue positivo el hemocultivo y en los tres restantes se aisló de LCR, pus, absceso y orina.

El diagnóstico de ingreso más frecuente fue el de Síndrome Diarreico Agudo. La hospitalización alcanzó un promedio de 28,7 días, la alteración de las deposiciones a 17,6 y la fiebre se mantuvo por 5,5 días. En 31 casos $(33,3 \%)$ el origen fue intrahospitalario. La diarrea se presentó en el $100 \%$ de los casos, siendo en el 32,3\% francamente enterocólica. La fiebre se dio en el $81,7 \%$ y la deshidratación en et $62,4 \%$. La hepatomegalia y esplenomegalia fueron poco frecuentes ( $10,8 \%$ y $6,5 \%$ respectivamente).

EI recuento leucocitario fue normal en $87,5 \%$ de los casos. Hubo leucocitosis en $8,3 \%$ y leucopenia en $4,2 \%$. Se controló el empleo de antibióticos en 83 enfermos, recibiendo éstos un promedio de 2,8 en forma simultánea o sucesiva. Los más usados fueron la Gentamicina en 59 casos, la Sulfatrimetoprina en 49 y la Furoxona en 43 casos

La letalidad fue de $8,6 \%$ ( 8 casos), el $100 \%$ de los fallecidos era menor de 6 meses. De los 14 casos con formas generalizadas fallecieron $4(28,6 \%)$ y todos presentaron compromiso meníngeo.

\section{SUMMARY}

93 clinical charts of sucklines Hospitalized as from January 1973 until December 1978 at the Pediatric Service of the Leonardo Guzmán Hospital in Antofagasta, were analyzed under the diagnosis of salmonellosis. Those cases with Salmonella tiphy development were not included.

The highest rate was obtained in those under the age of six months, with 48 cases $(51,6 \%)$, and from six to eleven months 36 cases $(38,7 \%)$, what adds up to $90,3 \%$ under the age of one. No differences were observed regarding their nutritive state and there was a predominance of the male, with 67 . cases $(61,3 \%)$

In 46 infants only Salmonella sp. was identified without a major differentiation. 23 cases $(24,7 \%)$ correspond to Salmonella Enteritidis group B and 25 to the tiphymudium variety $(26.9 \%)$. Salmonella was discovered in the coproculture of 80 patients. In 10 cases the hemoculture was positive and in the 3 remaining ones, it was isolated from CRL, pus, absecess and urine.

The most frequent diagnosis on arrival was that of acute Diartheic Syndrome. Hospitaiization reached an average of 28.7 days, alteration of depositions 17.6 and fever was maintained throughout 5.5 days. In 31 cases (33.3\%) the disease was acquired within the hospital. Diarrhea was observed in a $100 \%$ of the cases, being enterocholical in a $32.3 \%$. Fever was detected in an $81.7 \%$ and Dehydration in a $62.4 \%$. Hepatomegalia and Splenomegalia were infrequent $(10.8 \%$ and $6.5 \%$ respectively $)$.

The leukocyte count was normal in an $87.5 \%$ of the cases. There was Leukocytosis in an $8.3 \%$ and Leukopenia in a $4.2 \%$.

The use of antibiotics was checked in 83 patients, each one receiving an average of 2.8 of them. The most commonly used wew Gentamycin in 59 cases, Sulfatrimetoprin in 49 and furoxone in 43 cases.

The death rate was of $8.6 \%$ ( 8 cases), a $100 \%$ of the deceased was under the age of 6 months. Of the 14 cases with generalized forms, 4 died $(28.6 \%)$ and they all showed meningeal involvement.

\section{REFERENCIAS}

1 Prenzel I., Duarte E. Bacteriología de las diarreas. Hosp. Roberto del Río. Pediatría 16:92, 1973.

2 Prado V.; Donoso, E.; Zilleruelo G. Infección enteral por Salmonella Typhimurium en lactantes y sensibilidad in vitro de este germen en 7 antibióticos. Rev. Chil. Pediatría. 42:91, 1971.

3 Prado V.; Mimica I.; Donoso, E. Etiología bacteriana de las diarreas agudas. Rev. Chil. Pediatria. 45: $135,1974$.

- Prenzel, I.; Duarte E. Vigilancia epidemiológica de las diarreas. Verano 1976-77. Hosp. Roberto del Río. Pediatría 21: 139, 1978.

5 Lynch, B.; Valenzuela, M.C.; Donoso, E.; Cofré, J.; Vilches, A.; Agüero, M.E. Estudio de sensibilidad de Enterobacterianas a Sulfametoxazol, Trimethoprim y Cotrimexazol. Rev. Chil. Pediatría 46:17, 1975.

b Prenzel, 1. Salmonella en localizaciones intra y extraintestinales. Bol. Inst. Bacteriológico 15:41, 1973. 
7 Zacarías, J.; Brink, P. Hepatitis por Salmonellas. Rev. Chil, Pediatría 45: 35, 1974.

* Rossettein, B. Salmoneliosis in infant and childrens. J. of Ped. 701:1, 1957.

- Goldemberg, E.; Macaya, J.; Besnier, L.; Calderón. A.; Duffau, G.; Emilfork, M. y Soriano, H. Análisis clínico de lactantes con Síndrome diarreco agudo con deshidratación a Salmonella Typhimurium. Pediatría 17:79, 1975.
10 Prado, V. Salmonellosis, aspectos clinicos y epidemiológicos. Rev. Chil. Pediatría 46:264, 1975.

11 Goldemberg, A.; Macaya, G. y cols. Análisis clínico de 100 niños en coprocultivo positivo a Salmonella Typhimurium. Bol. Inst. Bacteriológico 15:52, 1973.

12 Guerrero J. y Cassorla, R. Salmonellosis por Salmonella Newington. Rev. Chil. Pediatria 44: 129, 1973. 